\title{
Research and Implementation on the credibility of user behavior Based on behavior declaration
}

\author{
Yu Xuejun ${ }^{1, a}$, Lv Haigeng ${ }^{1, b}$, \\ ${ }^{1}$ Beijing University of Technology School of Software Engineering, Beijing, 100124, China \\ ayuxuejun@bjut.edu.cn, ${ }^{\mathrm{b}}$ Ihg9136@163.com, *
}

Keywords: user behavior, the credibility of software, behavior declaration

\begin{abstract}
In today's Internet era, as the Web site was attacked continuously, the credibility of the software shows more important. The credibility of the software mainly refers to the behavior of software at run time is consistent with the expected behavior, which is a special form of software quality. This paper is to study the credibility of user behavior in B/S mode, put forward a verification model of Web software, which validate the credibility of the user's behavior by combining with behavior declaration.
\end{abstract}

\section{Introduction}

With the high-speed development of Internet, the credibility of the information system is also growing, especially in security, reliability, availability, and so on several aspects. On the issue, the international organization TCPA/TCG developed hardware platform capable of safety, China has conducted a lot of research on this problem, and put forward the trusted platform control module (TPCM), on the basis of TPM increase control module, which is more flexible [1,2]. At the same time, with the scale enlargement of the software and the complexity of the internal structure, under the background of Internet, its credibility has become one of the key research problems. The credibility of the software in addition to focus on internal quality and external quality, also pay more attention to in the use level of quality and security. In the reference[3], the author according to the "With the quality of system as the core, taking the use quality as the research object", in order to guarantee the credibility of the complex software system, build a new software technology system under Internet environment. In addition, in the open environment, in order to ensure the security of the system in operation, build a reliable software system, studying the user's behavior can reduce the interaction with malicious users, which improve the credibility of the process of interaction between service providers and users.[4]. In this background, the domestic researchers use declaration to define software behavior, and connecting with the whole life cycle of software development, put forward the whole life-cycle process model of application credibility verification. In this model, the Behavior declaration to define, generate, install, quote, delete and other operations, so as to ensure the credibility of the application software[5].

This research mainly focuses on the method based on behavior declaration to verify the credibility of the software, in a Web application environment, based on HTTP protocol, behavior actions of the user requests and server responses the credibility of the test, so as to ensure the credibility of the Web application software.During the study, According to the credibility validation model, combining with behavior declaration, test the Web application software and form the test report.

The first part of this article introduces the definition and contents of the behavior declaration;The second part introduces the whole life-cycle process model of application credibility verification;The third part introduces application software validation of the model;The fourth part introduces the application platform model and application platform for example to illustrate the credibility of the B/S application software validation application methods. 


\section{Application behavior declaration}

\subsection{The definition and content of behavior declaration}

Behaviors Declaration refers to describe a collection of software behavior related to software reliability, includes only describe the expected behavior of the software, these descriptions are trusted Behavior Declaration the participant acknowledges that, as part of the trusted software.

Behaviors Declaration using XML format in this study, its definition content as shown in table 1 and the main contents are as follows:

a.User permissions filter, by distinguishing different user permissions to regulate the content of the behavior declaration, which can look for the relevant behavior in the Application Behavior Declaration Library easier.

b.The expiration time/response time, is mainly used to determine whether HTTP requests within the effective time of a response.

c.Certification request method, considering the HTTP request method in the request is different, the request method can be used to define the behavior subject request, for example, some behavior can only use the GET method for data request and cannot request using the POST method, which improve the resource security of the Web server.

d.Multiple rules, which is the main part of the behavior declaration, mainly defined the content level of security and the expected results.

\section{2 the whole life-cycle process model of application credibility verification}

To complete the application software credibility validation, this article refer to the whole life-cycle process model of application credibility verification[5], as shown in figure 1.Its major match "WORD" to "DEED", in the whole life cycle of software development, embedded declared behavior, under the background of behavior declaration, generation, installation, the definition of reference, and delete operations, through the study of the definition of Web application behavior declaration, generate the application behavior library, and in test run stage declared by calling behavior declaration, to verify whether the software behavior's "DEED" matches its "WORD", to verify the credibility of the application software.

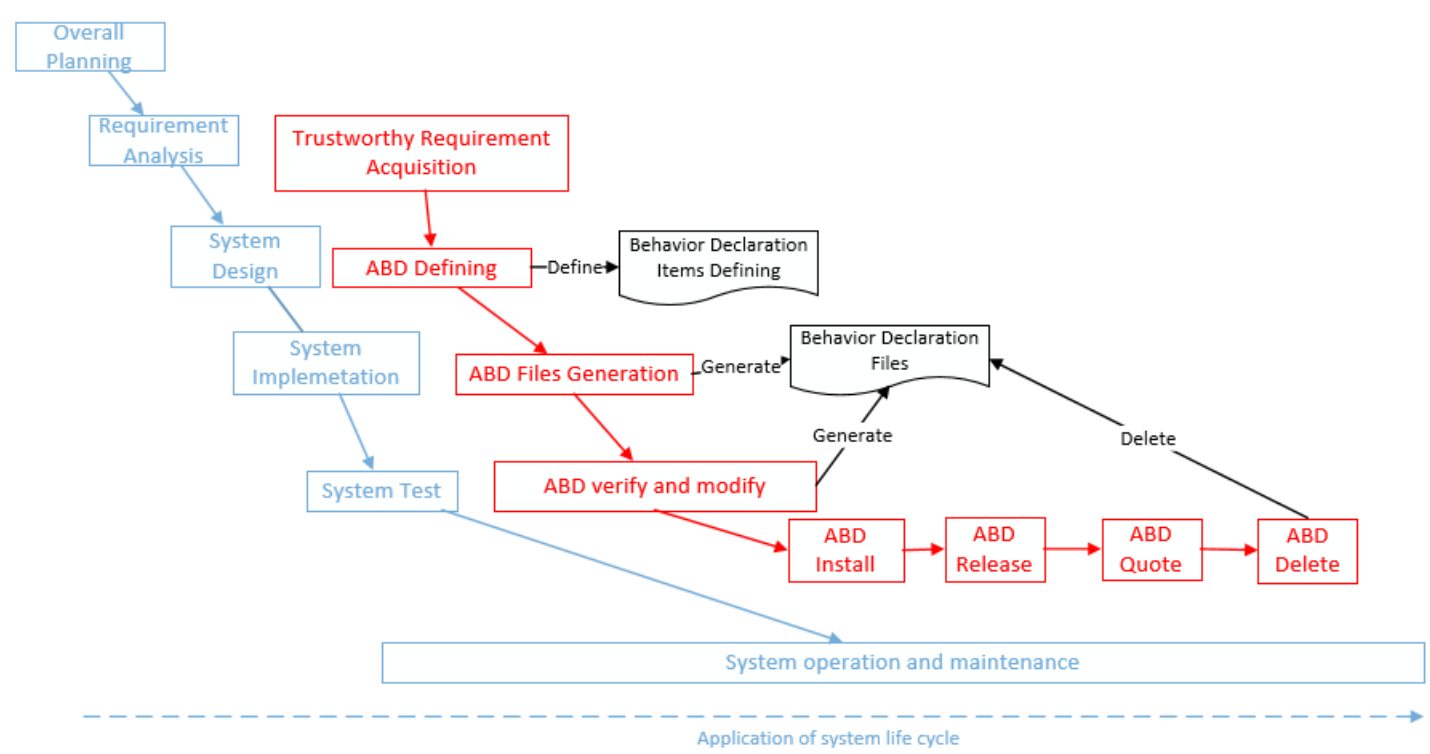

Fig.1 the whole life-cycle process model of application credibility verification

\section{Application software verification model}

Web application is mainly use the HTTP protocol to complete the information interaction, in the HTTP protocol, the Browser send the HTTP request to the server, the server receives the request processing, the processed results in the HTTP response pattern is returned to the Browser.HTTP 
requests mainly include the request line, request header and the request entity, mainly in the HTTP response contains a status line, response headers, and status codes. This research mainly through the access to the HTTP request and response in the body of the message, to analyze user behavior and the server response behavior, and complete the validation of Web application software reliability.

Research mainly in the Web application environment, in the HTTP request and response model, combined with behavior declaration formed a credibility validation of Web application software model, as shown in figure 2.

In figure 2 is shown in the Web application software credibility validation model, in which the user through the browser, sending HTTP requests, the adaptor will intercept this request, in turn, the following:

a.Controller analysis the request content, and determine its behavior, to get the behavior declaration from the ABDL by the content of the behavior.

b.ABDL according to the content of the request, return its behavior declaration to the controller.

c.Controller hand over the request behavior and and its behavior declaration to the judge module, to determine the behavior of the request.

d.According to parsing behavior declaration, judge module determine the content of the request behavior and return its results to the controller, which including the security level of the request behavior and its expected result.

e.If the security level of the request behavior is dangerous, controller will stop the request and submit its content and expected results to record module.If the security level of the request behavior is safety or suspicious, the controller will send the request to the Web server, Web server through HTTP requests for processing and interact with the database server, will return to the HTTP response control module to the HTTP response, parse the response, the response results and compare the request the expected results in operation d, If both agree, the HTTP response back to the user, if the two are inconsistent, controller can stop the HTTP response, and submit its content and expected results to record module, finally returned to the user the error report.

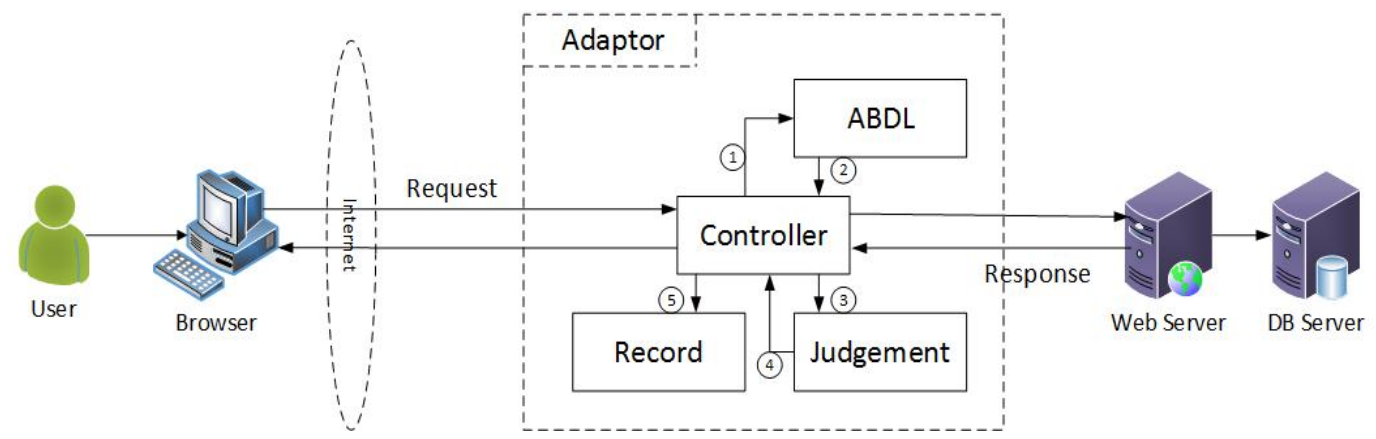

Fig.2 the verification model of Web software

The model is mainly based on two points to verify the credibility of requests, the request behavior is consistent with the definition of behavior declaration, the two refers to the response results are consistent with the expected results.Both of them are indispensable, only two are verified to determine the request behavior is credible, it records the requests and returns the error information back to the user.The main difference of this model and instrumentation method is that, this model validate the credibility of the request behavior before request the web server,which improve the filtering of risk behaviors, so as not to risk behaviors in the web server is malicious operation;In addition, this model also adds the response results and expected results, it equals to a credibility authentication server response content, which improve the credibility of user behavior in the process of the request and response.

\section{Application example of the verification model}

Behavior declaration definition, generation, installation, etc., to generate behavior declaration library, are accompanied by simultaneous Web application software development life cycle, so the define mechanism of the $\mathrm{ABD}$ is mainly based on software requirements, for example, application 
software users can upload resources in the Web environment, ABD should define the statement to upload resources, limit the property shall not exceed the scope of the definition of the threshold, this is functional requirement; Set of server response time, refers to the behavior declaration should to define the user request response time, If the response time exceeds the definition, it will automatically return the error report to the user, which is non functional requirements. After defining the $\mathrm{ABD}$, as the software development, with collecting these ABD files, form the library of ABD eventually.

In order to verify the model proposed in this article, and test flow chart, this article selects the web resource sharing platform as samples, the main function of samples including rights management, resource management, file upload and download, etc., after analysis the system requirement, determining to upload files is a sensitive behavior,so we validate its credibility in this example.

Based on the upload files operation, define its behavior declaration, which shown below.

$<$ PropertyList $>/ /$ Label

$<$ Authority $>$ User $</$ Authority $>/ /$ user permission: user

$<$ Timeout $>1000</$ Timeout $>/ /$ response time

$<$ Method $>$ POST $</$ Method $>/ /$ request method: POST

$<$ Property $>/ /$ a rule

$<$ Operation $>$ Upload_File $</$ Operation $>$ //upload file operation

$<$ Size $>4096</$ Size $>/ /$ file size

$<$ Type $>$ jpeg/png $<$ /Type $>/ /$ file type

$<$ SecurityLevel $>$ Safety $</$ SecurityLevel $>/ /$ security level : safety

$</$ Property $>$

$<$ Property $>/ /$ a rule

$<$ Operation $>$ Upload_File_Path $<$ /Operation $>/ /$ upload file operation

$<$ DisPath $>$ C: $\mid$ Users $\backslash$ Administrator $\mid$ Desktop $</$ DisPath $>/ /$ upload file path

$<$ SecurityLevel $>$ Suspicious $</$ SecurityLevel $>/ /$ security level : suspicious

$</$ Property $>$

$<$ PropertyList $>$

According to the $\mathrm{ABD}$ and the test cases, application testing platform test sample program, which test report as shown in table 1.

Table1 Test report

\begin{tabular}{llll}
\hline Permission & Operation & Content & Security level \\
\hline User & Upload_File & test.gif & Dangerous \\
User & Upload_File_Path & C:IUsers\Administrator\Desktop & Suspicious \\
\hline
\end{tabular}

According to the test report can be seen that the user upload file operations, file types of GIF, which is restricted inconsistent with the $A B D$, so the operation is defined in the system for dangerous operation in operation before the execution of the for block and risk behaviors were recorded; Another User operation Upload_File_Path, which path is C: \Users \Administrator \ Desktop, consistent with the ABD definition, therefore concluded that the operation is a suspicious behavior which is defined in the ABD.

In this paper, in addition to the tests according to verify the model and the flow chart for sample testing program, also has carried on the comparison with traditional instrumentation program, compare the results as shown in table 2, 3 .

Table2 Comparison of response time

\begin{tabular}{llll}
\hline Type & Operation & Content & Avg response time \\
\hline Instrumentation & Upload_File & test.gif & 231(ms) \\
verification mode & Upload_File & test.gif & $176(\mathrm{~ms})$ \\
Instrumentation & Upload_File_Path & C:IUsers\Administrator\Desktop & 278(ms) \\
verification mode & Upload_File_Path & C:IUsers\Administrator\Desktop & 273(ms) \\
\hline
\end{tabular}


Table3 Comparison of system operation's number

\begin{tabular}{llll}
\hline Type & request number & operate number & reason \\
\hline Instrumentation & 15 & 15 & $/$ \\
verification mode & 15 & 11 & danger operation \\
\hline
\end{tabular}

According to table 2, you can see the comparison of response time, when the operation is judged for dangerous, verification mode has obvious advantages on response time, when the operation is judged for suspicious, verification mode and traditional instrumentation authentication are not too big difference on the response time. Its reason according to table 3, you can see that under the same testing 15 different test cases, the number of execution system of instrumentation way to 15, and verification mode's number is 11, the remaining four judged dangerous operations which is prevented directly and recorded into the report.

\section{Conclusion}

This paper mainly aiming at validating credibility the software in web application environment, proposes a verification model of Web application under B/S mode based on HTTP protocol.This model mainly includes three parts, the first part is to obtain the content of the HTTP request and response, the second part is combined with behavior declaration verified the credibility of the content, the third part is to return the results to the user and record the results, through the operation to complete the credibility validation of user behavior.In addition,after comparing the model and the traditional method of instrumentation, found that the model can effectively stop dangerous operation, so as to ensure the credibility of the application software.

\section{Reference}

[1] Shen C X, Zhang H G, Feng G D.Overview of information security [J], Chinese Science Series E: Information Sciences(2007),37:129-150

[2]Zhang H G， Zhao B.Trusted Computing， Wuhan University Publishers (2011)

[3]Mei H , Cao D G. Software trustworthiness: the challenges brought by the Internet[J],Communications of the CCF, Vol. 6-2 (2010)

[4]Lin C, Tian L Q, Wang Y Z.Trusted network user behavior in the credible research[J], Research and development of the computer(2008), 45(2): 2033-2043

[5]Yu X J, Jiang G, Wang P.Research on application's credibility verification Based on ABD[J], Wuhan University Journal of Natural Sciences Volume21 Number1 February 2016 P.63-P.68 